Running Head: Gaze-cueing and Endogenous Attention

Gaze-cueing and endogenous attention operate in parallel

\author{
Gillian Slessor ${ }^{1}$ Ailbhe Finnerty ${ }^{1}$ Jessika Papp Daniel T. Smith ${ }^{2}$ Douglas Martin ${ }^{1}$ \\ University of Aberdeen ${ }^{1}$ \\ Durham University ${ }^{2}$
}

Word count: 7304

Address Correspondence To:

Gillian Slessor

School of Psychology

University of Aberdeen

Kings College

Aberdeen, AB24 3FX

UK

Email: gillian.slessor@abdn.ac.uk 


\begin{abstract}
The present research assessed the nature of endogenous shifts of attention based on internally generated expectations (i.e., target location probability) and involuntary attention shifts following eye-gaze cues from line-drawings of schematic faces (Experiment 1) and photographs of real neutral faces (Experiment 2) and fearful faces (Experiment 3). The timecourse of these two forms of attention was explored by manipulating the gaze-target SOA (i.e., $100 \mathrm{~ms}, 200 \mathrm{~ms}, 300 \mathrm{~ms}$ ). In all three experiments, target location probability influenced responding at each SOA with faster responses to high probability than low probability targets. However, the time-course of involuntary attention shifts was dependent on the gaze-cueing stimulus employed. For photographs of neutral gaze, endogenous orienting of attention was most efficient at the briefest SOA with involuntary attention shifts emerging later. However, both schematic and fearful gaze-cues influenced responding across all SOAs, which is indicative of stronger gaze-cueing effects from these cues. At $200 \mathrm{~ms}$ there was an additive effect as responses were slowest when the target had been invalidly cued by neutral gaze and also appeared in the low probability location. Taken together these findings suggest that these forms of involuntary and endogenous attention can operate in parallel and relatively independently, but can show potentially differing levels of influence, dependent on the time course in which they take to operate.
\end{abstract}

Key words: Gaze-cueing, Endogenous Attention, Target Location Probability 


\section{Gaze-cueing and endogenous attention operate in parallel}

Gaze following refers to the rapid allocation of attention in response to observing a shift in the eye-gaze direction of another person. In the lab, these effects are operationalized in gaze-cueing tasks, in which an observer is presented with a face showing averted gaze and is required to make some form of perceptual judgment about a target that appears at the gazed-at location or at some other location. Although the effects have been widely studied there is conflicting evidence regarding the nature of attention shifts in gaze-cueing and as a consequence the mechanisms underlying gaze-cueing remain contentious (e.g., Nummenmaa \& Calder, 2009).

There is considerable evidence that gaze-cueing occurs rapidly, and apparently involuntarily, which is suggestive of the engagement of an involuntary attention system (e.g., Driver et al., 1999; Friesen \& Kingstone, 1998; Friesen, Ristic, \& Kingstone, 2004). However, neuropsychological studies have demonstrated a double dissociation between gazecueing and exogenous attention. For instance, Vecera and Rizzo (2006) found that while gaze-cueing was disrupted, exogenous orienting to peripheral cues was preserved in a patient with a frontal lobe lesion. In contrast, gaze-cueing is preserved and exogenous attention disrupted in healthy participants who are unable to plan eye-movements (Smith, Schenk, \& Rorden, 2012). Furthermore, unlike abrupt peripheral cues, inhibition of return (IOR; delay in RT to targets appearing in a previously cued location) has either not been observed in gazecueing studies (Friesen \& Kingstone, 2003a, 2003b), emerged much later than in peripheral cueing (Frischen, Smilek, Eastwood \& Tipper, 2006) or has only been found under certain conditions (Frischen et al., 2006; Okamoto-Barth \& Kawai, 2006). For example, OkamotoBarth and Kawai (2006) found evidence of IOR only when they included catch trials in their gaze cueing paradigm. They argued that at longer SOAs participants disengaged from the cue as they expected it to be a catch trial and thus, unlike typical IOR seen in peripheral cueing 
studies, this effect was mediated by endogenous mechanisms. Importantly, Friesen and Kingstone (2003b) found that peripheral cue-induced IOR can co-exist with gaze-cueing effects, occurring at the same SOA but different locations. They argued that these findings are indicative of the two processes being independent. Taken together these findings demonstrate that gaze-cueing does not engage the same processes as exogenous attention.

An alternative view is that gaze-cueing engages the same endogenous attentional mechanism as symbolic cueing. For example, recent neurophysiological studies of gazecueing have shown that symbolic cues such as texture cues that are assigned a specific directional meaning, and gaze-cues elicit similar patterns of neural activation (although the gaze-cues produced a more rapid shift of attention; Brignani, Guzzon, Marzi, \& Miniussi, 2009; Chanon \& Hopfinger, 2011). However, these results simply demonstrate an association between gaze-cueing and endogenous attention, and do not offer strong evidence that gaze-cueing utilizes the same cognitive mechanism as endogenous attention. Furthermore, similar to the findings for exogenous cueing, other studies have indicated that there is a double dissociation between endogenous attention and gaze-cueing. For instance, neglect patients have been found to show intact gaze-cueing but disrupted arrow-cueing (Vuilleumier, 2002), while those with schizophrenia and patients with damage to the temporal lobes have specific difficulties with gaze-cueing but show intact arrow-cueing and can orient attention in response to pointing cues (Akiyama et al., 2006; 2008; Dalmaso, Galfano, Tarqui, Forti, \& Castelli, 2013 but see also Langdon, Seymour, Williams \& Ward, 2017). Gaze and arrow-cueing tasks also elicit different ERP waveforms (Hietanen, Leppanen, Nummenmaa, \& Astikainen, 2008). Unfortunately, it has been argued that arrowcueing itself is a poor measure of endogenous attention, as arrows can result in rapid and involuntary shifts of attention, which has been attributed to the directional meaning of these cues being overlearned in daily life (Brignani et al., 2009). Consequently, studies that directly 
compare arrows and gaze without using a purely symbolic cue can offer only weak evidence regarding the role of endogenous attention in gaze-cueing.

Further investigations of the role of endogenous mechanisms of attention in gazecueing have explored orienting of attention to counter-predictive gaze-cues (Friesen, et al., 2004; Kingstone, Friesen, \& Gazzaniga, 2000; Tipples, 2008). For example, in their work on gaze-cueing Friesen and colleagues elegantly demonstrate that while attention is involuntarily oriented to schematic face eye-gaze cues at very brief SOAs (i.e., $105 \mathrm{~ms}$ ), even when targets appear infrequently at such locations, no such effects occur for endogenous cues (i.e., when participants have the expectation that eye-gaze is predominantly counter-predictive). Therefore, at brief SOAs involuntary gaze-cues dominate the orientation of attention even when perceivers have been given instructions that should lead them to endogenously focus on an alternative location. At longer SOAs (i.e., $1200 \mathrm{~ms}, 1800 \mathrm{~ms}$ ), however, they show that attention can be successfully endogenously oriented towards the likely location of the target but not to locations cued by eye-gaze.

In a further instruction manipulation, Galfano et al. (2012) explored whether gazecueing could be suppressed when participants were told with $100 \%$ certainty about subsequent target location. Either before each trial (experiment 1a) or prior to each block of trials (experiment 2a) participants were given a direction word cue (e.g., left or right) and explicitly told that this cue was $100 \%$ predictive of the target location in the next trial (experiment 1a) or throughout the whole block of trials (experiment 2a). They were also told that the gaze-cues were distractors and should be ignored. Across both experiments participants still showed interference effects from the gaze-cues, responding more slowly when gaze direction was incongruent with target location. This gaze cueing effect occurred at both short and long SOAs, indicating that distracting gaze information still influenced 
attention even when participants had time to ignore it. According to Galfano et al. (2012) these findings suggest that gaze cueing is strongly automatic.

To date, behavioral experiments examining the interaction between gaze-cueing and endogenous shifts of attention have tended to do so only by comparing performance associated with an external cue (i.e., an arrow-cue, word-cue or the gaze-cue itself). While this has proved a highly fruitful and informative strategy to follow, one which has illuminated how attention operates in many situations, it only represents a single form of endogenous attention. An alternative way to explicitly test the hypothesis that gaze-cueing does not engage endogenous attention is to place gaze-cues in direct competition with a mechanism of endogenous attention that is driven not by an external cue but instead by prior experience and expectations.

One robust method of engaging endogenous attention without using symbolic cueing is to manipulate the task context such that participants have a sustained bias towards a particular spatial location (e.g., Chun \& Jiang 1998). Studies manipulating this endogenous attention shift have found that target location probability produces an attentional bias with targets in higher probability locations being detected more quickly than those in less likely locations (Chun \& Jiang, 1998; Fiser \& Aslin, 2001; Geng \& Behrmann, 2002; 2005). Research has also assessed the interaction between this and other attentional cues, such as centrally presented arrows or peripheral flashes of light (Geng \& Behrmann, 2005). As expected target detection was faster when the target appeared in a high probability location compared to a low probability location suggesting that target location probability was influencing visual processing. Attentional shifts were also found for centrally presented arrow cues with responses being quicker to valid than invalid trials. Importantly, there was no interaction between these two attentional shifts (target location probability and arrows) indicating that they were operating independently. In a further experiment an interaction was 
found between the attention orienting to target probability location and peripheral flashes of light. Here the magnitude of the cueing effect from the peripheral cue was weakest in the condition where target location probability was high. According to Geng and Behrmann (2005) these results suggest that target location probability produces the strongest attentional shift.

The critical result here is that arrow cues provided an attentional enhancement that was independent of the bias generated by task context, whereas the efficacy of the peripheral cues was mediated by the task context. In other words, an endogenous attentional bias towards one spatial location affected exogenous cueing but did not affect arrow cueing. This result is consistent with behavioural studies showing that exogenous attention can be modulated by top-down attentional control settings (Folk, Remington, \& Johnston, 1992).

Here, we investigate the extent to which gaze-cueing interacts with top-down endogenous attentional control. If gaze-cueing does not draw on the same cognitive mechanism as internally generated forms of endogenous attention the effects of the probability manipulation should not interact with the effects of non-predictive gaze-cueing. However, if both forms of attention do not operate independently, then attention shifts to gaze cues will be mediated by target location probability.

\section{Experiment 1}

In Experiment 1 we examined the role of endogenous attention in non-predictive gaze-cueing. Endogenous attention was manipulated independently of eye-gaze using a probabilistic expectancy of target location. Specifically, dependent on the experimental condition, targets appeared at an expected location in $66 \%$ (i.e., high probability), $50 \%$ (i.e., random probability) or $34 \%$ (i.e., low probability) of trials. For example, in a single block targets could have appeared on the left side of visual space on $66 \%$ of trials and on the right side of visual space on $34 \%$ of trials, thereby engendering an expectancy of where targets 
are likely or unlikely to appear. Importantly, this design meant that on any given trial the most reliable indicator of target location was the endogenously driven probabilistic expectancy of where targets were likely to appear.

\section{Method}

\section{Participants and Design}

Thirty-three undergraduates ( 24 female) from the University of Aberdeen completed the experiment for course credit. The experiment had a 3 (Target Location: high probability, random probability, low probability) X 3 (SOA: $100 \mathrm{~ms}, 200 \mathrm{~ms}, 300 \mathrm{~ms}$ ) x 2 (Trial Type: valid or invalid) repeated measures design. Two participants were excluded because of excessively long reaction times (> 3 S.D. above the median of all participants).

\section{Stimulus materials and Procedure}

Participants arrived at the laboratory and were seated in front of a PC at a viewing distance of approximately $57 \mathrm{~cm}$. Prior to completing the experiment all participants gave informed consent. Participants were instructed that they would be taking part in an experiment examining the ability to ignore irrelevant distracting information. The gaze-cue stimuli consisted of images of schematic faces portraying neutral expressions ( 313 x 337 pixels). Gaze direction of these images was manipulated creating one image with gaze directed to the left and one with gaze to the right. Target stimuli were asterisks $(*)$ presented in Arial size 24 font.

Participants were informed that they would see images of schematic faces in the centre of the screen followed by asterisks appearing to the left or right side of these faces. It was explained that the faces were to be ignored distracter stimuli and that the task was simply to respond as quickly and accurately as possible to the location of the target, pressing $\mathrm{Z}$ if it appeared on the left and $\mathrm{M}$ if it appeared on the right. Each trial comprised the presentation of a fixation cross (Arial font, size 24) for $500 \mathrm{~ms}$, this was followed by the 
gaze-cue stimulus, which was presented either for $100 \mathrm{~ms}, 200 \mathrm{~ms}$ or $300 \mathrm{~ms}$. Immediately following the disappearance of the gaze-cue, a target asterisk appeared on the left or right side of where the gaze-cue appeared and remained on the screen until a response was made (see Figure 1 for an example of a trial sequence). The inter trial interval was $1000 \mathrm{~ms}$. The order of presentation of gaze-cues and target locations was randomized. The order of blocks was counterbalanced across participants. The PC recorded the accuracy and latency of each response.

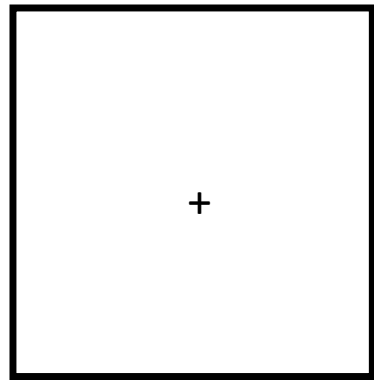

$500 \mathrm{~ms}$

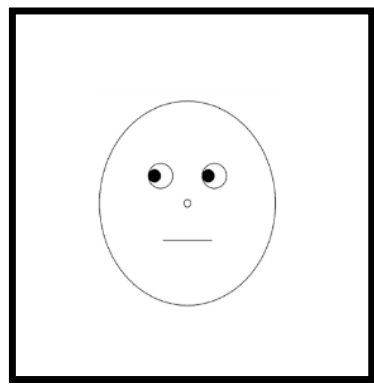

100,200 or $300 \mathrm{~ms}$

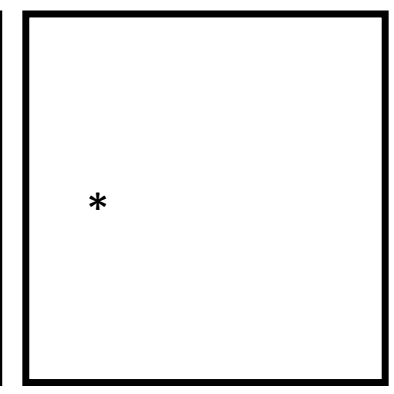

Until Response

Time

Figure 1. Example of a trial sequence in the gaze-cueing task. In the trial illustrated the target (the asterisk) appears on the congruent side.

The experiment was divided into three blocks of 144 trials. The probability of targets appearing in a certain location was manipulated across blocks, such that in one block targets appeared in the same location in $66 \%$ and $34 \%$ of trials (e.g., 96 targets appeared on the left and 48 targets appeared on the right), in one block targets appeared in the same location in $50 \%$ of trials (i.e., 72 targets appeared on the left and 72 targets appeared on the right), and in one block targets appeared in the same location in $34 \%$ and $66 \%$ of trials (e.g., 48 targets appeared on the left and 96 targets appeared on the right). Participants were not explicitly informed about the probability of the targets appearing in a certain location in each block. 
Eye-gaze cues looked left and right on an equal number of trials in each block (i.e., 72 leftward gazes and 72 rightward gazes). In half of the trials direction of the eye-gaze cues was congruent with target location.

\section{Results}

The dependent measure of interest was the median reaction time with which target locations were identified (see Figure 2). Trials on which errors were made (3\%) were excluded from the analysis. A 3 (Target Location: high probability, random probability, low probability) X 3 (SOA: $100 \mathrm{~ms}, 200 \mathrm{~ms}, 300 \mathrm{~ms}$ ) X 2 (Trial Type: valid or invalid) repeated measures ANOVA was undertaken on the data. In all analyses, Bonferroni pairwise comparisons were used to follow up significant main effects. The analysis revealed a main effect of Target Location $\left[F(2,64)=29.11, p<.001 ; \eta p^{2}=.476\right]$, with participants faster to identify targets appearing at high probability locations $(M=301 \mathrm{~ms})$ than either neutral probability locations $(M=309 \mathrm{~ms})$ or low probability locations $(M=319 \mathrm{~ms})$; participants were also faster to identify targets appearing at neutral probability locations than at low probability locations. There was also a main effect of SOA $\left[F(2,64)=119.77, p<.001 ; \eta p^{2}\right.$ $=.789$ ], with participants responding faster to targets when the SOA between gaze-cue and target presentation was $300 \mathrm{~ms}(M=300 \mathrm{~ms})$ relative to both $200 \mathrm{~ms}(M=307 \mathrm{~ms})$ and 100 ms $(M=322 \mathrm{~ms})$; participants were also faster to respond when the SOA was $200 \mathrm{~ms}$ relative to $100 \mathrm{~ms}$. There was also a main effect of Trial Type $[F(1,32)=132.69, p<.001$; $\left.\eta \mathrm{p}^{2}=.806\right]$, with participants faster to respond to valid than invalid trials (respective $M s=$ $299 \mathrm{~ms}$ vs. $320 \mathrm{~ms})$. There was also evidence of SOA X Trial Type interaction $[F(2,64)=$ $\left.18.65, p=.001 ; \eta p^{2}=.368\right]$. No other two- or three-way interactions were found to be significant [Target Location $\mathrm{x}$ SOA, $F(4,128)=1.05, p=.39, \eta \mathrm{p}^{2}=.032$; Target Location $\mathrm{x}$ Trial Type, $F(2,64)=1.58 p=.22, \eta p^{2}=.047$; Target Location $x$ SOA x Trial Type, $F(4$, $128)<1]$. 
(A)

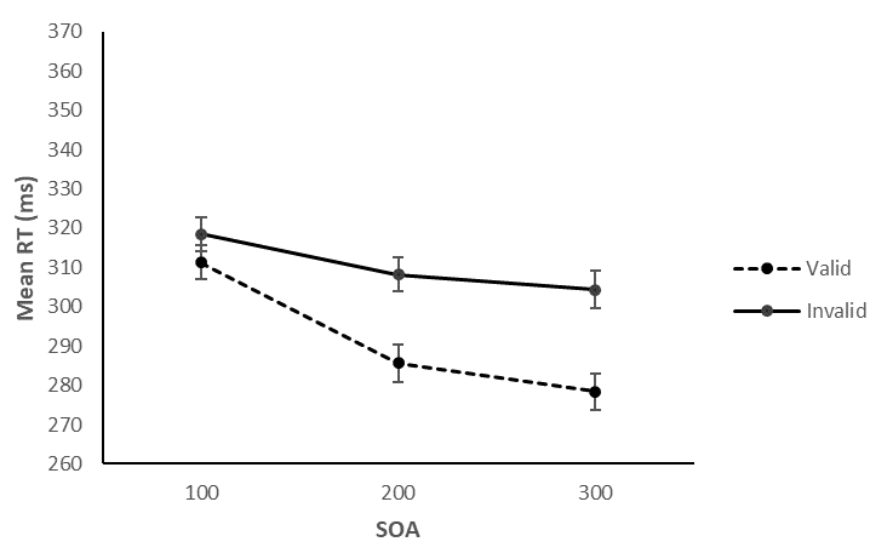

(B)

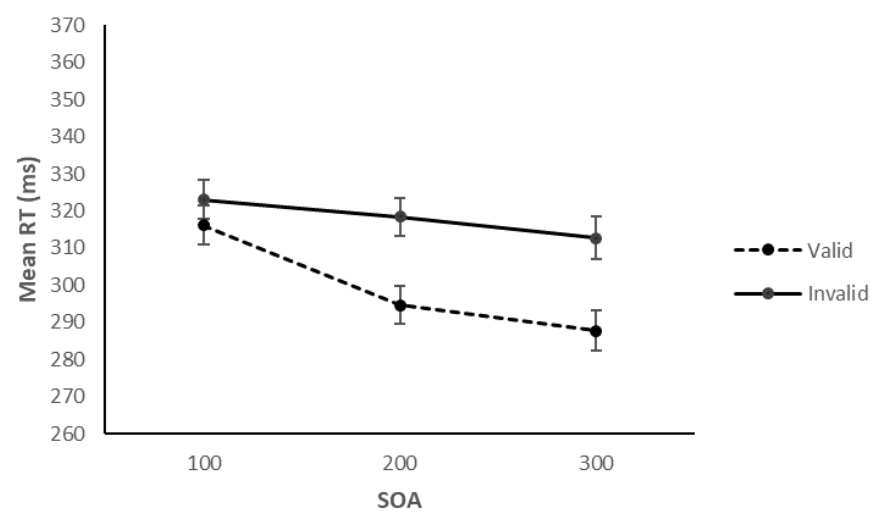

(C)

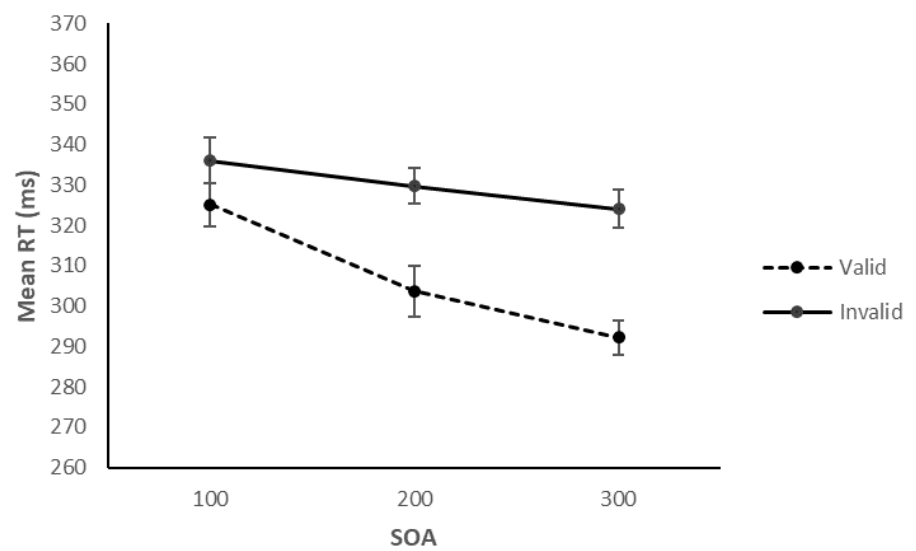

Figure 2. Mean Reaction Times (ms) for valid and invalid trials on the gaze-cueing task with schematic faces when the target was in the (A) high, (B) random and (C) low probability location. Error bars show +/-1 SEM. 
To further examine the interaction between SOA and Trial Type post-hoc paired samples t-tests were conducted comparing valid and invalid trials at each SOA. The results revealed that participants were faster to respond to valid trials than invalid trials at all SOAs [100 ms condition, $t(32)=4.84, p<.001 ; 200 \mathrm{~ms}$ condition, $t(32)=8.51, p<.001$ and 300 ms condition, $t(32)=9.03, p<.001]$.

Difference scores (invalid RT minus valid RT collapsed across all three target location probabilities) were then calculated to assess the strength of the gaze-cueing effect in each SOA condition. Paired sample t-tests revealed that the strength of the gaze-cueing effect was greater for $300 \mathrm{~ms}(M=28 \mathrm{~ms})$ relative to $100 \mathrm{~ms}(M=8 \mathrm{~ms}), t(34)=5.38, \mathrm{p}<$ .001 . The gaze-cueing effect was also significantly stronger at $200 \mathrm{~ms}(M=24 \mathrm{~ms})$ compared to $100 \mathrm{~ms}, t(32)=4.64, \mathrm{p}<.001$. There was no difference in the strength of the gaze-cueing effect between SOAs of $200 \mathrm{~ms}$ and $300 \mathrm{~ms}, t(32)=1.11, p=.274$.

\section{Discussion}

The data are indicative of overall endogenous cueing with participants responding faster to targets appearing at likely locations and slower to targets that appeared in unlikely locations. Therefore endogenous attention was indexed by a shift away from unlikely target locations and towards likely target locations. Gaze-cueing was also evident at all SOAs such that participants responded more quickly to targets that appeared at the gazed-at location. Critically, there was no interaction between gaze-cue validity and the probable location of the target. Specifically, participants experienced the same involuntary gazecueing effect at both attended and unattended locations. These data demonstrate that topdown, endogenous attention control and involuntary gaze-cueing can operate in parallel and do not draw on the same cognitive mechanism. 


\section{Experiment 2}

The results from Experiment 1 suggest that both endogenous and involuntary shifts of attention can occur and persist independently of one another even at relatively brief SOAs and within the context of a single processing encounter. There was no evidence that one form of attention was entirely dominant at any point (i.e., the presence of one form of attention shift in the absence of the other) nor was there evidence of a shift from one form of attention to another over time (i.e., an increase in one form of attention accompanied by a decrease in the other). This finding is perhaps unsurprising given that previous research indicates that target location probability and gaze-cues from schematic faces follow a similar initial time-course (Friesen et al., 2004; Geng \& Behrmann, 2005). It is possible that any differences in the influence of endogenous and involuntary attention would only be apparent when the onset time of each attentional shift differs; we explored this possibility in Experiment 2.

In order to further examine the interaction over time between the effects of target location probability and shifts of attention driven by gaze-cues we repeated the task used in Experiment 1, but using photographs of faces as the involuntary cue instead of schematic faces. Importantly, findings of previous research suggest that the time-course for extracting information from photographic and schematic faces differs. For example, people are quicker and more accurate at decoding emotional facial expressions from schematic (vs. real) faces (Kendall, Raffaelli, Kingstone, \& Todd, 2016). In addition, the majority of studies which have found evidence of gaze-cueing at short SOAs of around 100ms have used schematic faces (Friesen \& Kingstone, 1998; Friesen et al., 2004; Friesen \& Kingstone, 2003b).

Studies using real faces have found that stronger and more reliable gaze-cueing effects do not emerge until around 200 to 300 ms (Bayliss, Di Pellegrino, \& Tipper, 2005; Driver et al., 1999; Langdon et al., 2017). Research comparing gaze-cueing effects of schematic and 
real faces at 200ms SOA found that schematic gaze-cues resulted in stronger gaze-cueing effects than real faces (Hietanen \& Leppanen, 2003). They argued that these enhanced cueing effects for schematic faces might be due to differences in the complexity of the images as schematic faces do not contain additional noise (e.g., to skin texture). According to Risko, Laidlaw, Freeth, Foulsham, \& Kingstone (2012) enhanced attention orienting to schematic gaze cues might also be due to non-social mechanisms such as motion detection. We hypothesised that, as in Experiment 1, target location probability will trigger an early endogenous shift of attention that will persist at all SOAs. As shifts of attention from photographs of gaze-cues do not emerge until later (i.e., after $200 \mathrm{~ms}$ ) we predicted that involuntary shifts of attention would only occur when the SOA was sufficiently long to allow the extraction of eye-gaze information.

\section{Method}

\section{Participants and Design}

Twenty-six undergraduates (19 Female) from the University of Aberdeen completed the experiment for course credit. The experiment had a 3 (Target Location: high probability, random probability, low probability) X 3 (SOA: $100 \mathrm{~ms}, 200 \mathrm{~ms}, 300 \mathrm{~ms}$ ) x 2 (Trial Type: valid or invalid) repeated measures design.

\section{Stimulus materials and Procedure}

The materials and procedure for Experiment 2 were identical to those for Experiment 1 apart from one key difference; whereas in Experiment 1 schematic face images were used as the centrally presented cue, in Experiment 2 photographs of faces with eyes looking to the left or right were employed. The gaze-cue stimuli comprised grayscale digital headshot images of 4 unfamiliar people ( 2 female $\& 2$ male) with neutral expressions; for each identity there was an image of the person looking to the right and an image of them looking 
to the left (i.e., there were 8 eye-gaze images in total). The order of blocks was counterbalanced across participants.

\section{Results}

The dependent measure of interest was the median reaction time with which target locations were identified (see Figure 3 ). Trials on which errors were made (2\%) were excluded from the reaction time analysis. A 3 (Target Location: high probability, random probability, low probability) X 3 (SOA: $100 \mathrm{~ms}, 200 \mathrm{~ms}, 300 \mathrm{~ms}$ ) X 2 (Trial Type: valid or invalid) repeated measures analysis of variance (ANOVA) was undertaken on the data. In all analyses, Bonferroni pairwise comparisons were used to follow up significant main effects. The analysis revealed a main effect of Target Location $[F(2,50)=15.17, p<.001$; $\left.\eta \mathrm{p}^{2}=.378\right]$, with participants faster to identify targets appearing at high $(M=321 \mathrm{~ms})$ and random probability locations $(M=324 \mathrm{~ms})$ than targets appearing at low probability locations $(M=337 \mathrm{~ms})$; there was no difference in response time between targets appearing at high and random probability locations. There was also a main effect of SOA $[F(2,50)=$ 43.42, $\left.p<.001 ; \eta p^{2}=.635\right]$, with participants responding faster to targets when the SOA between gaze-cue and target presentation was $300 \mathrm{~ms}(M=319 \mathrm{~ms})$ relative to both $200 \mathrm{~ms}$ $(M=325 \mathrm{~ms})$ and $100 \mathrm{~ms}(M=338 \mathrm{~ms})$; participants were also faster to respond when the SOA was $200 \mathrm{~ms}$ relative to $100 \mathrm{~ms}$. There was also a main effect of Trial Type $[F(1,25)=$ 79.38, $\left.p<.001 ; \eta \mathrm{p}^{2}=.760\right]$, with participants faster to respond to valid than invalid trials (respective $M s=322 \mathrm{~ms}$ vs. $333 \mathrm{~ms}$ ). There was evidence of an SOA X Trial Type interaction $\left[F(2,50)=11.08, p=.001 ; \eta p^{2}=.307\right]$ and the Target Location $X$ Trial Type interaction approached significance $\left[F(2,50)=2.96, p=.061 ; \eta p^{2}=.106\right]$. All of these effects were subsumed in a Target Location X SOA X Trial Type interaction $[F(4,100)=$ 3.02, $p<.05 ; \eta p^{2}=.108$; see Figure 3]. 
(A)

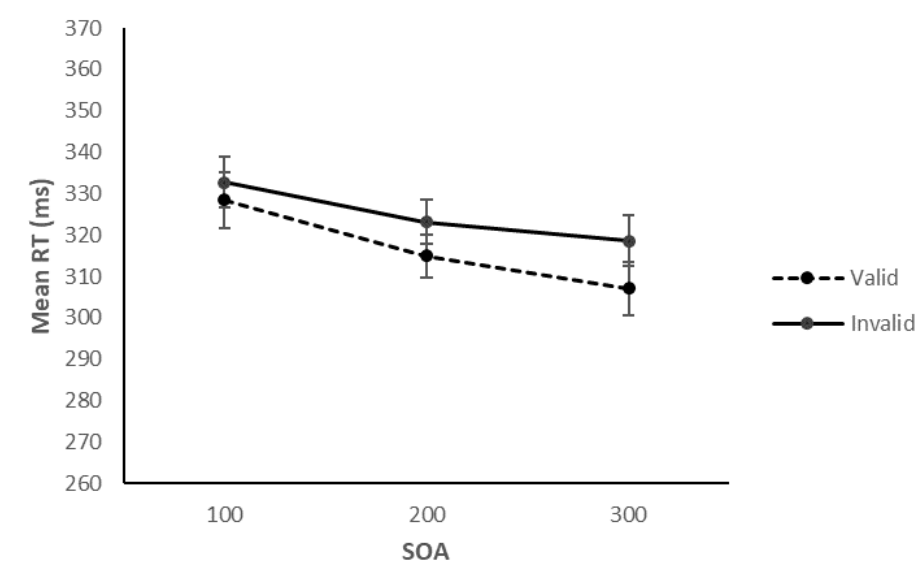

(B)

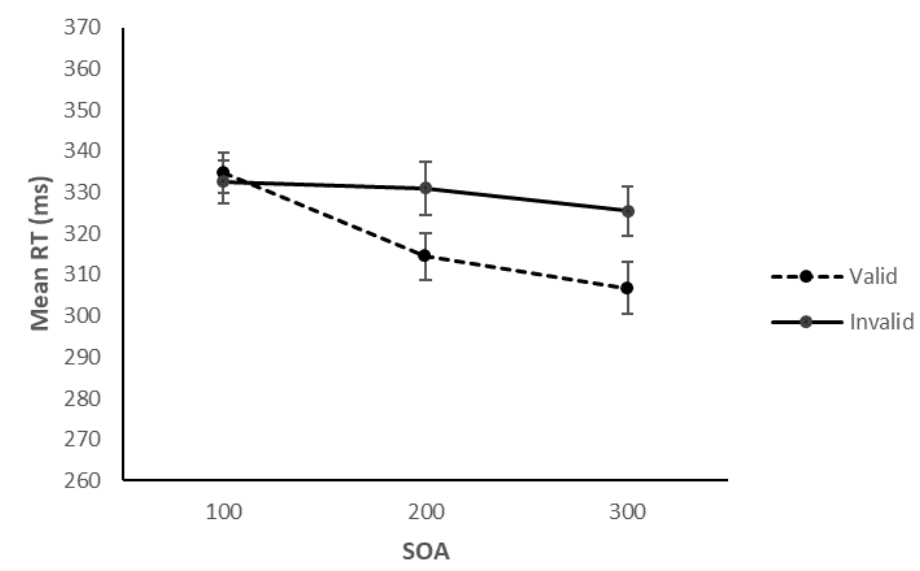

(C)

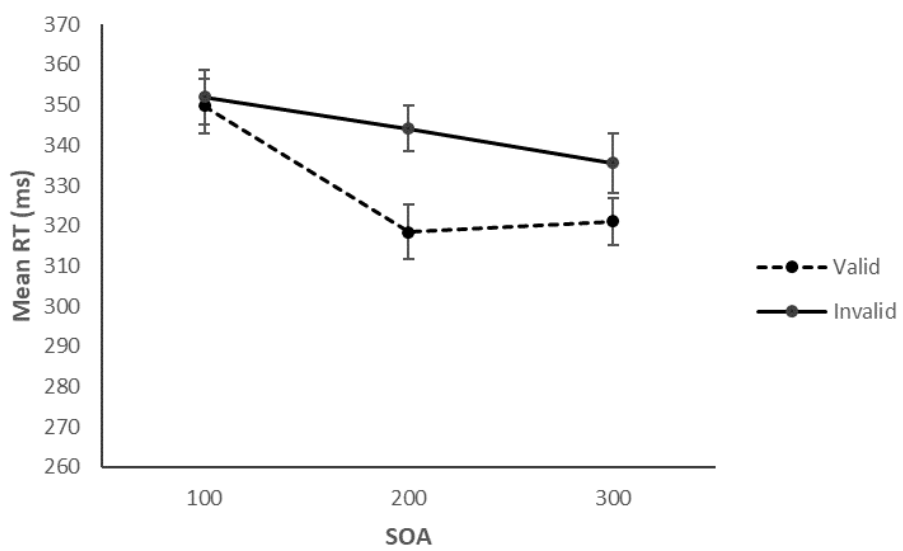

Figure 3. Mean Reaction Times (ms) for valid and invalid trials on the gaze-cueing task with photographs of neutral faces when targets were in the (A) high, (B) random and (C) low probability locations. Error bars show +/-1 SEM. 
To further explore this interaction separate 3 (Target Location: high probability, random probability, low probability) X 2 (Trial Type: valid or invalid) repeated measures ANOVAs were conducted on the reaction time data from each SOA. In all analyses, Bonferroni pairwise comparisons were used to follow up significant main effects. Analysis of the $100 \mathrm{~ms}$ condition revealed a main effect of Target Location $[F(2,50)=14.00, p<$ $\left..001 ; \eta p^{2}=.359\right]$, with participants faster to identify targets in high $(M=331 \mathrm{~ms})$ and random probability locations $(M=334 \mathrm{~ms})$ than targets in low probability locations $(M=$ $351 \mathrm{~ms}$ ); there was no difference in response time between targets appearing at high and random probability locations. There was no evidence of a main effect of Trial Type $[F(1$, $\left.25)<1, p=.50 ; \eta p^{2}=.019\right]$ nor of a Target Location $X$ Trial Type interaction $[F(2,50)=$ $\left.1.19, p=.31 ; \eta p^{2}=.045\right]$.

Analysis of the $200 \mathrm{~ms}$ condition revealed a main effect of Target Location $[F(2,50)$ $\left.=6.61, p<.01 ; \eta p^{2}=.209\right]$, with participants faster to identify high probability targets $(M=$ $319 \mathrm{~ms})$ than low probability targets $(M=331 \mathrm{~ms})$; there was no difference in response time between high and random probability conditions $(M=323 \mathrm{~ms})$, nor between low and random probability conditions. There was also a main effect of Trial Type $[F(1,25)=$ 49.21, $p<.001 ; \eta p^{2}=.663$ ], with participants faster to respond to valid than invalid trials (respective $M s=316 \mathrm{~ms}$ vs. $333 \mathrm{~ms}$ ). These effects were subsumed within a Target Location X Trial Type interaction $\left[F(2,50)=6.66, p<.01 ; \eta p^{2}=.210\right]$. To further explore this interaction a series of paired samples t-tests were carried out on gaze-cued difference scores (i.e., invalid - valid) to compare the strength of the gaze-cue effect at each Target Location. Significantly smaller gaze-cueing effects were found in the high probability condition $(M=8 \mathrm{~ms})$ compared to the low probability condition $(M=26 \mathrm{~ms}), t(27)=3.19$, $\mathrm{p}<.01$. There was no difference in the magnitude of gaze-cueing between the random $(M=$ 
$17 \mathrm{~ms}$ ) and low probability, $\mathrm{t}(27)=1.13, \mathrm{p}=.269$, and high probability locations, $t(27)=$ $2.19, \mathrm{p}<.05$.

Analysis of the $300 \mathrm{~ms}$ condition revealed a main effect of Target Location $[F(2,50)$ $\left.=8.80, p<.01 ; \eta \mathrm{p}^{2}=.260\right]$, with participants faster to identify targets in high $(M=313 \mathrm{~ms})$ and random probability locations $(M=316 \mathrm{~ms})$ than those in the low probability location $(M=328 \mathrm{~ms})$; there was no difference in response time between targets appearing at high and random probability locations. There was also a main effect of Trial Type $[F(1,25)=$ $30.92, p<.001 ; \eta p^{2}=.553$ ], with participants faster to respond to valid than invalid trials (respective $M s=312 \mathrm{~ms}$ vs. $327 \mathrm{~ms}$ ). There was no evidence of a Target Location X Trial Type interaction $\left[F(2,50)<1, p=.344 ; \eta p^{2}=.042\right]$.

\section{Discussion}

The results of Experiment 2 suggest that, as predicted, relative to schematic faces, gaze-cueing emerges later when using photographs of faces. Consistent with the findings of Experiment 1, endogenous cueing of attention was evident at all SOAs such that participants were slower to respond to targets that appeared in a low probability location relative to those appearing in the high and random probability locations. However, the influence of the gaze-cue was dependent on the time interval between gaze-cue and target presentation. At SOAs of $100 \mathrm{~ms}$ gaze direction did not exert an effect on responses and therefore the endogenous attention shift emerged more quickly and, at brief SOAs, operated more efficiently than attention shifts in response to gaze-cues. However, at longer SOAs (i.e., 200 and $300 \mathrm{~ms}$ ) responses were also influenced by the gaze-cue such that participants responded more quickly to trials in which gaze direction validly predicted target location compared to invalid trials.

Of particular interest is the finding that at $200 \mathrm{~ms}$ the gaze-cueing effect was greatest when the target appeared in the low target location probability condition. Geng and 
Behrmann (2005) argued that if one attentional mechanism is stronger than another then the effect of the weaker cue will only emerge when the stronger cue is invalid. Therefore, this finding might suggest that in the $200 \mathrm{~ms}$ condition the endogenous shift still operates more efficiently than gaze, possibly because participants have had limited time to process the eyegaze direction and, as such, this cue is just beginning to influence attention.

The increase in the strength of the gaze-cueing effect when the target was in the low probability location was mainly driven by longer RTs in response to invalid gaze-cues, with similar reaction times for valid trials across all three target location probabilities (see Figure 3). This result is likely due to an additive effect, as in this condition both target location probability and gaze-cues were misdirecting participants, with the target appearing in the low probability location and being preceded by an invalid gaze-cue. Therefore, participants would have a strong expectation about where the target would appear and when their expectations were violated by the target appearing on the opposite side of the screen it would thus take longer to prepare and execute a response. The emergence of shifts of attention from eye-gaze cues at $200 \mathrm{~ms}$ could be evidence of the exogenous system, triggered by the sudden onset of external stimuli (i.e., eye-gaze), acting as a "circuitbreaker" by interrupting endogenous shifts of attention (Corbetta \& Schulman, 2005). Here, even when participants had built up an internal expectation of where targets were likely to appear based on target location probability, their attention was involuntarily captured by the sudden appearance of eye-gaze cues which made it increasingly difficult to detect invalidly cued targets.

Gaze-cues continued to influence responses at $300 \mathrm{~ms}$ with quicker responses to valid relative to invalid trials. Endogenous shifts of attention were also evident here and both attention mechanisms influenced visual processing to the same extent suggesting that at longer SOAs gaze direction had now been efficiently processed. 
Taken together the results of Experiment 2 suggest that target location probability and gaze-cues can have an independent and additive influence on target detection. These findings are consistent with those of Bockler, van der Wel, \& Welsh $(2014 ; 2015)$ who found that two different attention mechanisms (i.e., social or direct gaze and non-social motion cues) worked independently and in parallel, having an additive effect on attentional capture.

\section{Experiment 3}

The results of Experiment 2 suggest that, when photographs of real eye-gaze cues were used as the attention cue, endogenous shifts of attention emerged more quickly than involuntary gaze-cueing. In contrast, involuntary gaze-cueing has not been found to override endogenous attention mechanisms under any of the conditions in the previous two experiments. However, in both experiments the gaze-cueing image displayed a neutral expression, which is important as previous gaze-cueing research suggests that the emotional expression of the gaze cueing stimuli can influence responding. Specifically, fearful gaze cues have been found to result in stronger gaze-cueing effects than those displaying neutral expressions (Tipples, 2006) or other emotions such as happiness (Putman, Hermans, \& van Honk, 2006; Fichtenholtz, Hopfinger, Graham, Detwiler, \& Labar, 2007), arguably because fearful gaze signals the presence of threat in the environment and is thus processed more quickly and efficiently. Due to this enhanced cueing effect, it is possible that involuntary orienting of attention in response to fearful gaze cues may override endogenous orienting mechanisms such as internally generated expectations of target location.

To the best of our knowledge research has yet to explore the role that emotion plays in the interaction between involuntary attention shifts in response to gaze and endogenous control of attention. To address this issue the current experiment will examine how shifting attention in response to fearful gaze direction interacts with participants' internally generated 
expectations of target location probability. The task used in Experiment 2 was repeated but the photographs of faces used as the gaze cue portrayed fearful, rather than neutral, expressions. It is predicted that enhanced gaze-cueing in response to fearful gaze would lead to involuntary orienting of attention to gaze dominating over endogenous attention shifts based on target location probability.

\section{Method}

\section{Participants}

Thirty-six undergraduates (23 Female) from the University of Aberdeen completed the experiment for course credit. The experiment had a 3 (Target Location: high probability, random probability, low probability) X 3 (SOA: $100 \mathrm{~ms}, 200 \mathrm{~ms}, 300 \mathrm{~ms}$ ) x 2 (Trial Type: valid or invalid) repeated measures design.

\section{Stimulus materials and Procedure}

The materials and procedure for Experiment 3 were identical to those for Experiment 2 apart from one key difference; whereas in Experiment 2 photographs of neutral faces were used as the centrally presented cue, in Experiment 3 photographs of fearful faces with eyes looking to the left or right were employed. The gaze-cue stimuli comprised grayscale digital headshot images of 4 unfamiliar people ( 2 female \& 2 male) portraying fearful expressions. These images were taken from the Facial Expressions of Emotion: Stimuli and Test (FEEST) database (Young, Perrett, Calder, Sprengelmeyer, \& Ekman, 2002). For each identity there was an image of the person looking to the right and an image of them looking to the left (i.e., there were 8 eye-gaze images in total). The order of blocks was counterbalanced across participants.

\section{Results}


The dependent measure of interest was the median reaction time with which target locations were identified (see Figure 4). Trials on which errors were made (3.74\%) were excluded from the reaction time analysis. A 3 (Target Location: high probability, random probability, low probability) X 3 (SOA: $100 \mathrm{~ms}, 200 \mathrm{~ms}, 300 \mathrm{~ms}$ ) X 2 (Trial Type: valid or invalid) repeated measure ANOVA was carried out on the data. The analysis revealed a main effect of Target Location $\left[F(2,70)=16.22, p<.001, \eta p^{2}=.317\right]$, with participants faster to identify targets appearing at high $(M=313 \mathrm{~ms})$ and random probability locations $(M=322 \mathrm{~ms})$ than targets appearing at low probability locations $(M=333 \mathrm{~ms})$. In turn they were significantly quicker at responding to targets appearing at high than random probability locations. There was also a main effect of $\operatorname{SOA}\left[F(2,70)=59.96, p<.001, \eta p^{2}=\right.$ .627], with participants responding faster to targets when the SOA between gaze-cue and target presentation was $300 \mathrm{~ms}(M=314 \mathrm{~ms})$ relative to both $200 \mathrm{~ms}(M=321 \mathrm{~ms})$ and 100 $\mathrm{ms}(M=333 \mathrm{~ms})$; participants were also faster to respond when the SOA was $200 \mathrm{~ms}$ relative to $100 \mathrm{~ms}$. There was also a main effect of Trial Type $[F(1.35)=71.59, p<.001$, $\left.\eta p^{2}=.672\right]$, with participants faster to respond to valid than invalid trials (respective $M s=$ $316 \mathrm{~ms}$ vs. $330 \mathrm{~ms})$. There was evidence of an SOA X Trial Type interaction $[F(2,70)=$ $\left.13.84, p<.001 ; \eta p^{2}=.283\right]$. No other two- or three-way interactions were found to be significant [Target Location $\mathrm{x}$ SOA, $F(2.98,104.35)=.32, p=.808 \eta p^{2}=.009$; Target Location x Trial Type, $F(1.27,44.36)<1$; Target Location x SOA x Trial Type, $F(2,70)<$ $1]$. 
(A)

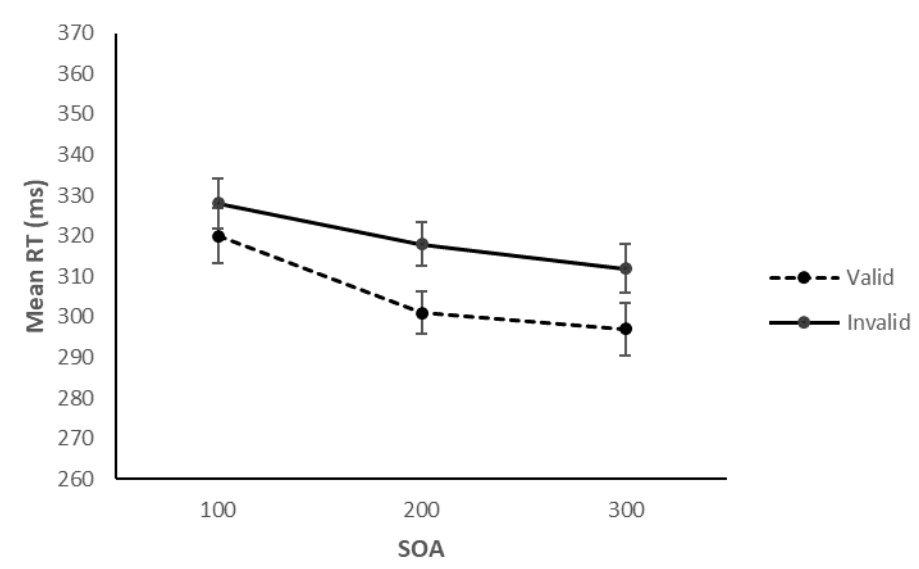

(B)

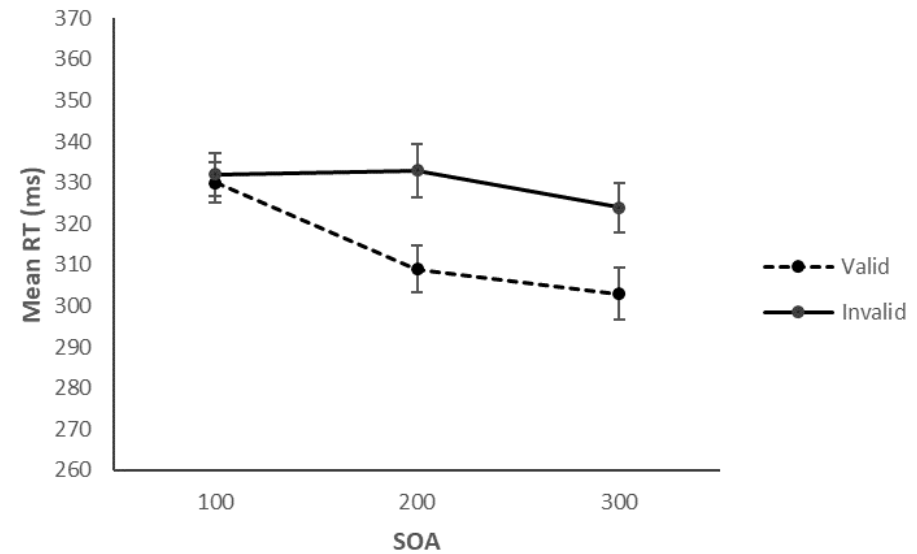

(C)

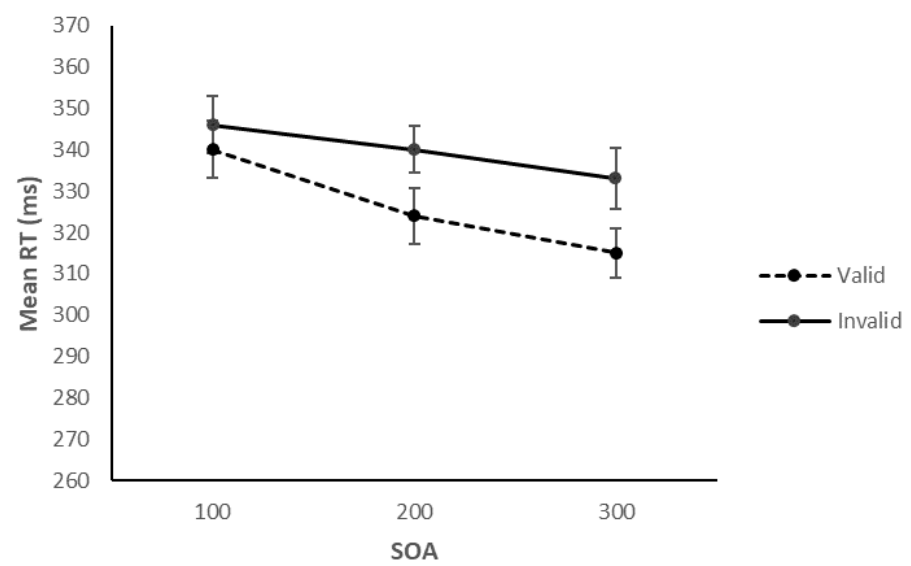

Figure 4. Mean Reaction Times (ms) for valid and invalid trials on the gaze-cueing task with photographs of fearful faces when targets were in the (A) high, (B) random and (C) low probability locations. Error bars show +/-1 SEM. 
In order to further explore the interaction between Trial Type and SOA, post-hoc paired-samples t-tests were conducted to compare valid and invalid trials at each SOA. The results showed that participants responded with shorter RTs to valid trials across all SOAs: $100 \mathrm{~ms}(M=5.19, S D=12.2), t(35)=2.56, p<.05, d=.43 ; 200 \mathrm{~ms}(M=19.1, S D=15.9)$, $t(35)=7.21, p<.001, d=1.20 ; 300 \mathrm{~ms}(M=18.0, S D=14.5), t(35)=7.44, p<.001, d=$ 1.24 .

Similar to the previous experiments difference scores (invalid RT minus valid RT) were then calculated in order to examine the strength of the gaze-cueing effect for each SOA. Paired sample t-tests revealed that the strength of the gaze-cueing effect was greater for $300 \mathrm{~ms}(M=18 \mathrm{~ms})$ relative to $100 \mathrm{~ms}(M=5 \mathrm{~ms}), t(35)=4.07, \mathrm{p}<.001$. The gazecueing effect was also significantly stronger at $200 \mathrm{~ms}(M=19 \mathrm{~ms})$ compared to $100 \mathrm{~ms}$, $t(35)=5.04, \mathrm{p}<.001$. There was no difference in the strength of the gaze-cueing effect between SOAs of $200 \mathrm{~ms}$ and $300 \mathrm{~ms}, t(35)=.40, p=.690$.

\section{Discussion}

Results of Experiment 3 indicated that, like the previous experiments, participants were making endogenous shifts of attention based on their internally generated expectations of target location, with responses being faster to targets in high probability locations and slowest to those in low probability locations. There was also evidence of involuntary attention cueing in that participants responded more quickly to gazed-at targets. Importantly, both endogenous and involuntary attention shifts were evident across all SOAs and there was no interaction between these attention mechanisms suggesting that they were operating independently and in parallel with both having a similar influence on responding. Therefore, contrary to predictions and previous findings that fearful gaze enhances gaze-cueing effects (Tipples, 2006), involuntary attention shifts in response to fearful gaze cues did not dominate or override endogenous attention mechanisms. However, it should be noted that previous 
research exploring the effects of emotional expression on eye-gaze cueing have been mixed. Hietatenen and Leppanen (2003) found that the emotion displayed did not influence gaze cueing, while other studies have found that it is dependent on the characteristics of the participant (Fox, Matthews, Yiend, \& Calder, 2007).

\section{General Discussion}

These studies aimed to explore the interaction between gaze-cueing and endogenous attentional mechanisms based on target location probability. Experiment 1 and 3 showed that when gaze was cued by a schematic and fearful face, respectively, gaze-cueing and endogenous attention operated in parallel with both influencing responding to a similar extent. In Experiment 2, when a photograph of neutral gaze was used, endogenous attention emerged first and was more efficient at directing attention at short SOAs, while involuntary attention shifts did not emerge until later. In addition, it was found that neutral gaze-cues and endogenous attention shifts had an additive effect on target detection but only during one SOA. These findings suggest that endogenous attention and gaze-cueing can occur independently and can show potentially differing levels of influence, dependent on the time course in which they take to operate.

Across all three experiments target location probability consistently influenced orienting of attention. Therefore, it seems that experiencing the relative probability of target location leads people to build up an expectation of where the targets are or are not likely to appear, which in turn causes endogenous biases in attention to visual space (Geng \& Behrmann, 2005). At the same time, the sudden occurrence of a potentially biologically relevant event, such as the presence of a gaze-cue, has the ability to involuntarily direct attention (Driver et al., 1999; Friesen \& Kingstone, 1998). Crucially, however, findings across the three experiments suggest that the time-course of involuntary shifts of attention was influenced by the type of gaze-cueing stimuli employed. 
The findings from Experiment 1 suggested that endogenous shifts of attention, based on target location probability, and schematic gaze-cues can operate together and in parallel. Here, both gaze-cues and target location probability influenced speed of target detection such that participants were faster to respond to targets that appeared in valid/high probability locations than invalid/low probability locations. These effects were present across all SOAs and there was no evidence of an interaction between the two forms of attention suggesting that they operated relatively independently of one another. These findings are in keeping with those assessing the relationship between target location probability and a directional arrow cue (Geng \& Behrmann, 2005). We argue that this finding is reflective of the attentional shifts produced by both gaze-cues and target location probability following a similar time course. For example, attentional shifts from both target location probability and schematic gaze-cues emerge at very brief SOAs (Friesen \& Kingstone, 1998; Geng \& Behrmann, 2005).

In support of this suggestion, a different pattern of results was revealed in Experiment 2 when the onset time of attentional shifts from target location probability and gaze-cue (i.e., gaze direction of photographs of neutral faces) differed. At an SOA of $100 \mathrm{~ms}$ endogenous shifts of attention emerged, indexed by a shift away from targets appearing in unlikely locations, while gaze direction did not influence responding. This discrepancy in the relative influence from gaze-cues of schematic relative to photographs of neutral faces can be explained by taking into account the time required to extract eye-gaze information from each stimulus. Specifically, social information has been found to be extracted more quickly from schematic than real faces (Kendall et al., 2016) and schematic gaze-cues reliably produces attentional shifts at 100ms (Friesen \& Kingstone, 1998: Friesen et al., 2004; Friesen \& Kingstone, 2003b), while photographs of faces do not (Driver et al., 1999; Bayliss, et al., 2005, Langdon et al., 2017). Therefore, viewing photographs of faces for less 
than $200 \mathrm{~ms}$ will not be sufficient to allow gaze processing, while schematic gaze-cues, which are less complex, are processed more quickly and efficiently at very brief SOAs.

However, findings from Experiment 3 suggest that, when the gaze-cueing stimulus is portraying a fearful expression, it is possible for a photograph of a real face to produce involuntary attention shifts at very brief SOAs. Here, findings were more akin to those for schematic gaze cues with both fearful gaze-cues and endogenous attention influencing responding to a similar extent at all SOAs. Again, we argue that the discrepancy between the findings for photographs of real faces displaying neutral and fearful expressions is due to the time taken to process these images. Whereas, schematic gaze cues were processed more quickly because of their simplicity, fearful gaze is likely to be extracted at greater speed because it is more biologically relevant to the perceiver than neutral gaze, as it is signalling the presence of threat in the environment (Tipples, 2006). Therefore, although, contrary to predictions, involuntary orienting to fearful gaze cues did not dominate over endogenous attention control, the findings do suggest that fearful faces enhances gaze cueing effects at short SOAs, in a similar way to schematic faces. In order to directly compare the time course and strength of these different gaze-cues (i.e., schematic, neutral and fearful) a future study should use a within subjects design in which each participant completes a gaze-cueing task with all three cue types.

When participants had been given sufficient time to extract eye-gaze information from photographs of neutral faces (i.e., by around 200-300 ms; Driver et al., 1999) gaze direction also began to influence responses with faster detection of targets in valid (vs. invalid) trials. Interestingly at an SOA of $200 \mathrm{~ms}$, responses were slowest when the target appeared in the low probability location and participants were misdirected by the gaze-cues, suggesting that the two forms of attention had an additive effect on target detection. Again, these findings might reflect the two attentional mechanisms following a different time 
course. Specifically, at $200 \mathrm{~ms}$ the endogenous shift was still more efficiently enacted, while involuntary gaze-cueing took longer to build and manifest and thus only started to influence responding at $200 \mathrm{~ms}$. At longer SOAs, attention shifts in response to gaze cues became more efficient and thus both forms of attention then influenced attention to the same extent.

Taken together the current findings indicate that, whereas involuntary shifts of attention in response to gaze-cues were dependent on the stimulus employed, target location probability consistently influenced responses in all three experiments. However, it is possible that target location probability could have differing levels of influence within a block of trials. Specifically, in the current study participants were not explicitly informed about the probability of the target appearing on the left or right and thus expectancies of target location probability had to be learned over time. Therefore, while gaze-cues would influence attention shifts throughout the block, endogenous shifts might not emerge until later when expectancies have been learned. Future research should explicitly explore the time course of attention shifts to gaze-cues and target location probability across a trial block.

In sum, the present study was the first to investigate the interaction over time between involuntary shifts in attention from eye-gaze cues and endogenous attentional shifts based on internally generated expectations of target location. Findings suggest that endogenous attention and gaze-cueing can operate in parallel, with results indicating that they exert an independent influence on target detection. Furthermore, when the time course of the two mechanisms differ, endogenous attention and gaze-cueing can show differing levels of influence. 


\section{References}

Akiyama, T., Kato, M., Muramatsu, T., Maeda, T., Hara, T., \& Kashima, H. (2008). Gazetriggered orienting is reduced in chronic schizophrenia. Psychiatry Research, 158, 287-296. https://doi.org/10.1016/j.psychres.2006.12.004

Akiyama, T., Kato, M., Muramatsu, T., Saito, F., Umeda, S., \& Kashima, H. (2006). Gaze but not arrows: A dissociative impairment after right superior temporal gyrus damage. Neuropsychologia, 44, 1804-1810. https://doi.org/10.1016/ j.neuropsychologia.2006.03.007

Bayliss, A.P., di Pellegrino, G., \& Tipper, S.P. (2005). Sex differences in eye gaze and symbolic cueing of attention. Quarterly Journal of Experimental Psychology, 58, 631650. https://doi.org/ 10.1080/02724980443000124

Böckler, A., Robrecht, P.R.D., \& Welsh, T.N. (2014). Catching eyes: Effects of social and nonsocial cues on attention capture, Psychological Science, 25, 720-727. https://doi.org/10.1177/0956797613516147

Böckler, A., Robrecht, P.R.D., \& Welsh, T.N. (2015). Eyes only? Perceiving eye contact is neither sufficient nor necessary for attentional capture by face direction. Acta Psychologica, 160, 134-140. https://doi.org/10.1016/j.actpsy.2015.07.009

Brignani, D., Guzzon, D., Marzi, C. A., \& Miniussi, C. (2009). Attentional orienting induced by arrows and eye-gaze compared with an endogenous cue. Neuropsychologia, 47, 370-381. https://doi.org/10.1016/j.neuropsychologia.2008.09.011

Chanon, V. W., \& Hopfinger, J. B. (2011). ERPs reveal similar effects of social gaze orienting and voluntary attention, and distinguish each from reflexive attention. Attention Perception \& Psychophysics, 73, 2502-2513. https://doi.org/10.3758/s13414-011-0209-4

Chun, M.M., \& Jiang, Y. (1998). Contextual cueing: Implicit learning and memory of visual 
context guides spatial attention. Cognitive Psychology, 36, 28-71. https://doi.org/10.1006/cogp.1998.0681

Corbetta, M., \& Schulman, G.L. (2002). Control of goal-directed and stimulus-driven attention in the brain. Nature Reviews Neuroscience, 3, 201-215. https://doi.org/10.1038/nrn755

Dalmaso, M., Galfano, G., Tarqui, L., Forti, B., \& Castelli, L. (2013). Is social attention impaired in schizophrenia? Gaze, but not pointing gestures, is associated with spatial attention deficits. Neuropsychology, 27(5), 608-613. https://doi.org/10.1037/a0033518

Driver, J., Davis, G., Ricciardelli, P., Kidd, P., Maxwell, E., \& Baron-Cohen, S. (1999). Gaze perception triggers automatic visuo-spatial orienting in adults. Visual Cognition, 6, 509-540. https://doi.org/ 10.1080/135062899394920

Fiser, J., \& Aslin, R.N. (2001). Unsupervised statistical learning of higher-order spatial structures from visual scenes. Psychological Science, 12, 499-504. https://doi.org/10.1111/1467-9280.00392

Fichtenholtz, H. M., Hopfinger, J. B., Graham, R., Detwiler, J. M., \& LaBar, K. S. (2007). Happy and fearful emotion in cues and targets modulate event-related potential indices of gaze-directed attentional orienting. Social, Cognitive, and Affective Neuroscience, 2, 323-333. https://doi.org/ 10.1093/scan/nsm026

Folk, C. L., Remington, R. W., \& Johnston, J. C. (1992). Involuntary covert orienting is contingent on attentional control. Journal of Experimental Psychology: Human Perception and Performance, 18, 1030-1044. https://doi.org/10.1037/00961523.18.4.1030 
Fox, E., Mathews, A., Calder, A. J., \& Yiend, J. (2007). Anxiety and sensitivity to gaze direction in emotionally expressive faces. Emotion, 7(3), 478-486. https://doi.org/10.1037/1528-3542.7.3.478

Friesen, C.K., \& Kingstone, A. (1998). The eyes have it! Reflexive orienting is triggered by non-predictive gaze. Psychonomic Bulletin \& Review, 5, 490-495. https://doi.org/10.3758/BF03208827

Friesen, C.K, \& Kingstone, A. (2003a). Covert and overt orienting to gaze direction cues and the effects of fixation offset. NeuroReport, 14, 489-493. https://doi.org/ $10.1097 / 00001756-200303030-00039$

Friesen, C.K., \& Kingstone, A. (2003b). Abrupt onsets and gaze direction cues trigger independent reflexive attentional effects. Cognition, 87, B1-B10. https://doi.org/ 10.1016/S0010-0277(02)00181-6

Friesen, C.K., Ristic, J., \& Kingstone, A. (2004). Attentional effects of counter-predictive gaze and arrow cues. Journal of Experimental Psychology: Human perception and performance, 30, 319-329. https://doi.org/10.1037/0096-1523.30.2.319

Frischen, A., Smilek, D., Eastwood, J.D., \& Tipper, S.P. (2007). Inhibition of return in response to gaze cues: The role of time course and fixation cue. Visual Cognition, 15(8), 881-895. https://doi.org/ 10.1080/13506280601112493

Galfano, G., Dalmaso, M., Marzoli, D., Pavan, G., Coricelli, C., \& Castelli, L. (2012). Eye gaze cannot be ignored (but neither can arrows). The Quarterly Journal of Experimental Psychology, 65(10), 1895-1910. https://doi.org/10.1080/ 17470218.2012 .663765

Geng, J.J., \& Behrmann, M. (2002). Probability cueing of target location facilitates visual search implicitly in normal participants and participants with hemispatial neglect. Psychological Science, 13, 520-525. https://doi.org/10.1111/1467-9280.00491 
Geng, J.J., \& Behrmann, M. (2005). Spatial probability as an attentional cue in visual search. Perception and Psychophysics, 67, 1252-1268. https://doi.org/10.3758/BF03193557

Hietanen, J.K., \& Leppanen, J.M. (2003). Does facial expression affect attention orienting by gaze direction cues? Journal of Experimental Psychology: Human Perception and Performance, 29, 1228-1243. https://doi.org/10.1037/0096-1523.29.6.1228

Hietanen, J.K., Leppanen, J.M., Nummenmaa, L., \& Astikainen, P. (2008). Visuospatial attention shifts by gaze and arrow cues: An ERP study. Brain Research, 1215, 123 136. https://doi.org/ 10.1016/j.brainres.2008.03.091

Kendall, L.N., Raffaelli, Q., Kingstone, A., \& Todd, R.M. (2016). Iconic faces are not real faces: enhanced emotion detection and altered neural processing as faces become more iconic. Cognitive Research, 1, 19. https://doi.org/ 10.1186/s41235-016-0021-8

Kingstone, A., Friesen, C.K., Gazzaniga, M.S. (2000). Reflexive joint attention depends on lateralized cortical connections. Psychological Science, 11, 169-166. https://doi.org/10.1111/1467-9280.00232

Langdon, R., Seymour, K., Williams T., \& Ward, P.B. (2017). Automatic attentional orienting to other people's gaze in schizophrenia. Quarterly Journal of Experimental Psychology, 70, 1549-1558. https://doi.org/10.1080/17470218.2016.1192658

Nummenmaa, L., \& Calder, A. J. (2009). Neural mechanisms of social attention. Trends in Cognitive Sciences, 13, 135-143. https://doi.org/10.1016/j.tics.2008.12.006

Okamoto-Barth, S., \& Kawai, N. (2006). The role of attention in the facilitation effect and another "inhibition of return". Cognition, 101(3), B42-50. https://doi.org/10.1016/j.cognition.2005.11.002

Putman, P., Hermans, E., \& van Honk, J. (2006). Anxiety meets fear in the perception of dynamic expressive gaze. Emotion, 6. 94-102. https://doi.org/ 10.1037/15283542.6.1.94 
Risko, E.F., Laidlaw, K.E.W., Freeth, M., Foulsham, T., \& Kingstone, A. (2012). Social attention with real versus reel stimuli: toward an empirical approach to concerns about ecological validity. Frontiers of Human Neuroscience, 6, 143. https://doi.org/10.3389/fnhum.2012.00143

Smith, D. T., Schenk, T., \& Rorden, C. (2012). Saccade preparation is required for exogenous attention but not endogenous attention or IOR. Journal of Experimental Psychology: Human Perception and Performance, 38, 1438-1447. https://doi.org/ $10.1037 / \mathrm{a} 0027794$

Tipples, J. (2006). Fear and fearfulness potentiate automatic orienting to eye-gaze. Cognition and Emotion, 20, 309-320. https://doi.org/ 10.1080/02699930500405550

Tipples, J. (2008). Orienting to counterpredictive gaze and arrow cues. Perception and Psychophysics, 70, 77-87.

Vecera, S. P., \& Rizzo, M. (2006). Eye gaze does not produce reflexive shifts of attention: Evidence from frontal-lobe damage. Neuropsychologia, 44(1), 150-159. https://doi.org/10.1016/j.neuropsychologia.2005.04.010

Vuilleumier, P. (2002). Perceived gaze direction in faces and spatial attention: a study in patients with parietal damage and unilateral neglect. Neuropsychologia, 40(7), 1013 1026. https://doi.org/10.1016/S0028-3932(01)00153-1 